\title{
OPEN Strong association of type 2 diabetes with degenerative lumbar spine disorders
}

\author{
Chul-Hyun Park ${ }^{1}$, Kyoung-Bok Min², Jin-Young Min ${ }^{3}$, Du Hwan Kim4, Kyung Mook Seo ${ }^{4} \&$ \\ Don-Kyu Kim ${ }^{4}$
}

Tantalizing connections between type 2 diabetes and degenerative lumbar spine disorders have become increasingly evident. However, the association of type 2 diabetes with degenerative lumbar spine disorders remains unclear. We sought to clarify the association between type 2 diabetes and lumbar spine disorders using nationwide data in Korea. Furthermore, we explored the association of diabetes with the prevalence of spinal procedures. The data in this study was obtained from Korean health claim database. Between 2016 and 2019, totals of 479,680 diabetes and 479,680 age- and sexmatched control subjects were enrolled. Patients with diabetes had more likely to have degenerative lumbar spine disorders and spinal procedures than controls. Using multivariate-adjusted analysis, patients with diabetes were at increased risk of being concomitantly affected by lumbar disc disorder [adjusted odds ratio 1.11 (95\% confidence interval 1.10-1.12)], lumbar spondylotic radiculopathy [1.12 (1.11-1.13)], spondylolisthesis [1.05 (1.02-1.08)] and spinal stenosis [1.16 (1.15-1.18)], compared to controls. Furthermore, diabetic patients had an increased risk of undergoing lumbar spinal injection [1.13 (1.12-1.14)], laminectomy [1.19 (1.15-1.23)], and fusion surgery [1.35 (1.29-1.42)]. We demonstrated that type 2 diabetes was significantly associated with lumbar spine disorders and frequent spinal procedures. Our results suggest diabetes as a predisposing factor for lumbar spine disorders.

Low back pain is ranked first globally when it comes to attributing the cause of years lived with disability (YLDs). It is an increasingly major problem in an aging society ${ }^{1}$. Mechanical dysfunction accounts for up to $97 \%$ of low back pain diagnoses ${ }^{2}$. The major causes of mechanical lumbar spine disorders are degenerative intervertebral disc disorders, lumbar Spondylotic radiculopathy, spondylolisthesis and spinal stenosis, all part and parcel of a degenerative cascade of the lumbar spine ${ }^{3,4}$. Despite low back pain patients being highly prevalent the world over ${ }^{1}$, evidence of risk factors for the degenerative lumbar spine disorders remains lacking. For many years, systemic reviews and meta-analysis pointed to genetic factors, aging, obesity, smoking and occupational factors being the risk factors for low back pain ${ }^{5-9}$. However, it has been noted that degeneration of lumbar spine disorders can be accelerated by metabolic factors and a toxic environment ${ }^{10}$. Therefore, the associations of lumbar spine disorders with metabolic diseases such as diabetes mellitus needs to be established ${ }^{11-13}$.

Type 2 diabetes can lead to various complications involving major organs, and gives rise to degenerative changes of the organs ${ }^{14}$. Various pathological changes in spine structure such as loss of disc height, decreased vertebral bone mass, and endplate sclerosis are well documented in diabetic model which reflect similar degenerative findings in human intervertebral discs ${ }^{15-17}$. In clinical data, there is conflicting evidence with regards to a relationship between diabetes and lumbar spine disorders. A close relation between type 2 diabetes and low back pain was reported ${ }^{18-20}$. However, a few studies reported that type 2 diabetes was linked to degenerative lumbar spine disorders ${ }^{21,22}$. A recent twin cohort study found that there was no significant association between diabetes and lumbar disc disorder ${ }^{23}$. Therefore, it is necessary to confirm the associations between diabetes and degenerative lumbar spine disorders by a large-scaled, population-based study. Furthermore, to determine the impact of diabetes on the outcome of lumbar spine disorders, the prevalence of spinal procedures in diabetes patients should be investigated. To the best of our knowledge, a population-based study of the associations of

\footnotetext{
${ }^{1}$ Department of Physical and Rehabilitation Medicine, Kangbuk Samsung Hospital, Sungkyunkwan University School of Medicine, Seoul, Korea. ${ }^{2}$ Department of Preventive Medicine, College of Medicine, Seoul National University, Seoul, Korea. ${ }^{3}$ Veterans Medical Research Institute, Veterans Health Service Medical Center, Seoul, Korea. ${ }^{4}$ Department of Physical Medicine and Rehabilitation, College of Medicine, Chung-Ang University, Seoul, Korea. ${ }^{\circledR}$ email: donkim21@cau.ac.kr
} 


\begin{tabular}{|c|c|c|c|c|c|c|}
\hline \multirow[b]{2}{*}{$\begin{array}{l}\text { Baseline } \\
\text { characteristics }\end{array}$} & \multicolumn{2}{|c|}{ Unmatched subjects } & \multirow[b]{2}{*}{$p$ value } & \multicolumn{2}{|c|}{ Matched subjects } & \multirow[b]{2}{*}{$p$ value } \\
\hline & \begin{tabular}{|l|} 
Control \\
$(\mathrm{n}=2,282,304)$
\end{tabular} & $\begin{array}{l}\text { Diabetic patients } \\
(\mathrm{n}=479,680)\end{array}$ & & \begin{tabular}{|l|} 
Control \\
$(\mathrm{n}=479,680)$
\end{tabular} & $\begin{array}{l}\text { Diabetic patients } \\
(\mathrm{n}=479,680)\end{array}$ & \\
\hline \multicolumn{7}{|l|}{ Age (year) } \\
\hline $20-29$ & $314,477(13.8)$ & $3,273(0.7)$ & \multirow{6}{*}{$<0.0001$} & $3,273(0.7)$ & $3,273(0.7)$ & \multirow{6}{*}{1.0} \\
\hline $30-39$ & $410,357(18.0)$ & $12,965(2.7)$ & & $12,965(2.7)$ & $12,965(2.7)$ & \\
\hline $40-49$ & $416,885(18.3)$ & $40,290(8.4)$ & & $40,290(8.4)$ & $40,290(8.4)$ & \\
\hline $50-59$ & $464,584(20.4)$ & $101,035(21.1)$ & & $101,035(21.1)$ & $101,035(21.1)$ & \\
\hline $60-69$ & $315,449(13.8)$ & $128,728(26.8)$ & & $128,728(26.8)$ & $128,728(26.8)$ & \\
\hline$\geq 70$ & $360,552(15.7)$ & $193,389(40.3)$ & & $193,389(40.3)$ & $193,389(40.3)$ & \\
\hline \multicolumn{7}{|l|}{ Sex } \\
\hline Male & $997,275(43.7)$ & $240,759(50.2)$ & \multirow{2}{*}{$<0.0001$} & $240,759(50.2)$ & $240,759(50.2)$ & \multirow{2}{*}{1.0} \\
\hline Female & $1,285,029(56.3)$ & $238,921(49.8)$ & & \begin{tabular}{|l|}
$238,921(49.8)$ \\
\end{tabular} & $238,921(49.8)$ & \\
\hline \multicolumn{7}{|l|}{\begin{tabular}{|l} 
Type of beneficiary \\
\end{tabular}} \\
\hline Medical insurance & $2,171,213(95.1)$ & $425,153(88.6)$ & \multirow{2}{*}{$<0.0001$} & $444,842(92.7)$ & $425,153(88.6)$ & \multirow{2}{*}{$<0.0001$} \\
\hline Medical aid & $111,091(4.9)$ & $54,527(11.4)$ & & $34,838(7.3)$ & $54,527(11.4)$ & \\
\hline \multicolumn{7}{|l|}{ Hypertension } \\
\hline Yes & $604,615(73.5)$ & $345,527(73.9)$ & \multirow{2}{*}{$<0.0001$} & 227,737 (47.5) & $345,527(73.9)$ & \multirow{2}{*}{$<0.0001$} \\
\hline No & $1,677,689(26.5)$ & $125,153(26.1)$ & & $251,943(42.5)$ & $125,153(26.1)$ & \\
\hline \multicolumn{7}{|c|}{\begin{tabular}{|l|} 
Chronic kidney disease \\
\end{tabular}} \\
\hline Yes & $23,984(98.9)$ & $38,839(8.0)$ & \multirow{2}{*}{$<0.0001$} & $9,547(2.0)$ & $38,839(8.0)$ & \multirow{2}{*}{$<0.0001$} \\
\hline No & $2,258,320(1.1)$ & $440,841(92.0)$ & & $470,133(98.0)$ & $440,841(92.0)$ & \\
\hline \multicolumn{7}{|l|}{ Dyslipidemia } \\
\hline Yes & $144,498(6.3)$ & $98,099(20.5)$ & \multirow{2}{*}{$<0.0001$} & $47,260(9.9)$ & $98,099(20.5)$ & \multirow{2}{*}{$<0.0001$} \\
\hline No & $2,137,806(93.7)$ & $381,581(79.5)$ & & \begin{tabular}{|l|}
$432,420(90.1)$ \\
\end{tabular} & $381,581(79.5)$ & \\
\hline
\end{tabular}

Table 1. Baseline characteristics of patients with type 2 diabetes in unmatched and matched population-based controls. Data are presented as number (percentage, \%).

diabetes with degenerative lumbar spine disorders and the prevalence of undergoing spinal procedures has not yet been studied.

Therefore, this study investigated the association between type 2 diabetes mellitus and degenerative lumbar spine disorders using a nationwide, population-based data in Korea. Furthermore, we explored the impact of diabetes on the prevalence of lumbar spinal injection and surgical procedures.

\section{Results}

Baseline characteristics. Table 1 shows the baseline characteristics of patients with type 2 diabetes in unmatched and matched population-based controls. The study ultimately enrolled 479,680 subjects each, both for patients with diabetes as well as for age- and sex- matched control subjects. Thus, a final study population were 959,360 subjects (Fig. 1). In each group, 240,759 subjects $(50.2 \%)$ were male $(p=1.0)$. For the type of beneficiary and underlying diseases, the proportions of medical aid, hypertension, chronic kidney disease, and dyslipidemia were significantly higher in the diabetes group than in matched control group, respectively (all $p$ value $<0.0001)$.

Prevalence of lumbar spine disorders and spinal procedures. In diabetic patients, the prevalence of lumbar disc disorder and spondylotic radiculopathy were $17.2 \%$ and $21.2 \%$, respectively, showing markedly higher compared to the control group ( $p<0.0001 ;$ Fig. 2$)$. The diabetes group also exhibited a higher prevalence in lumbar spondylolisthesis $(2.8 \%)$, and spinal stenosis $(23.1 \%)$ than the control group, respectively $(p<0.0001)$.

The prevalence of lumbar spinal injection was significantly higher in the diabetes group than in the control group ( $13.9 \%$ vs. $8.6 \% ; p<0.0001$ ) (Fig. 3 ). Furthermore, the diabetes group had more lumbar laminectomy $(1.8 \%$ vs. $1.0 \% ; p<0.0001)$, and lumbar fusion surgery $(0.8 \%$ vs. $0.3 \% ; p<0.0001)$ than those of the control group, respectively.

Association of diabetes mellitus with lumbar spine disorders and spinal procedures. Patients with diabetes were at an increased risk of comorbid lumbar disc disorder (aOR 1.11, 95\% CI 1.10-1.12) and lumbar spondylotic radiculopathy (aOR 1.12, 95\% CI 1.11-1.13) compared with the control group, even after adjustments for hypertension, chronic kidney disease, dyslipidemia, and the type of beneficiary (Table 2). Furthermore, diabetic patients had a significantly higher risk of lumbar spondylolisthesis (aOR 1.05, 95\% CI 1.021.08 ) and spinal stenosis (aOR $1.16,95 \% \mathrm{CI} 1.15-1.18$ ), respectively.

Diabetic patients were at increased risk of undergoing lumbar spinal injection (aOR 1.13, 95\% CI 1.12-1.14), lumbar laminectomy (aOR 1.19, 95\% CI 1.15-1.23), and fusion surgery (aOR 1.35, 95\% CI 1.29-1.42) compared with the controls, respectively (Table 3). 
The participants (20-89 years old) from Health Insurance

Review and Assessment Service Database between

January 2016 and December 2018 (n=2,761,984)

1:1 Age \& sex matched

by propensity score method

\section{Final study population $(\mathrm{n}=\mathbf{9 5 9 , 3 6 0 )}$}

- Type 2 Diabetes Mellitus ( $\mathrm{n}=479,680)$

- Age- \& Sex- Matched Healthy Control $(n=479,680)$

Figure 1. Selection of the study population.

A

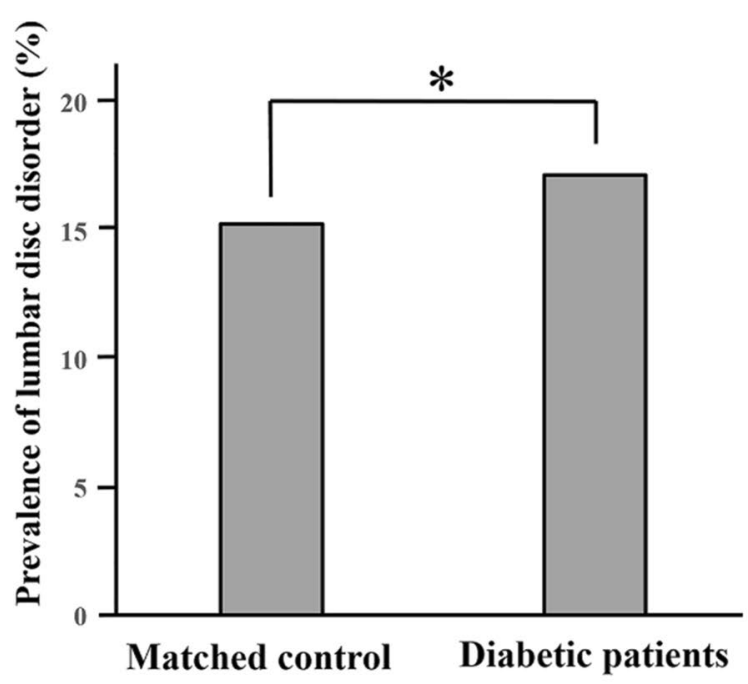

C

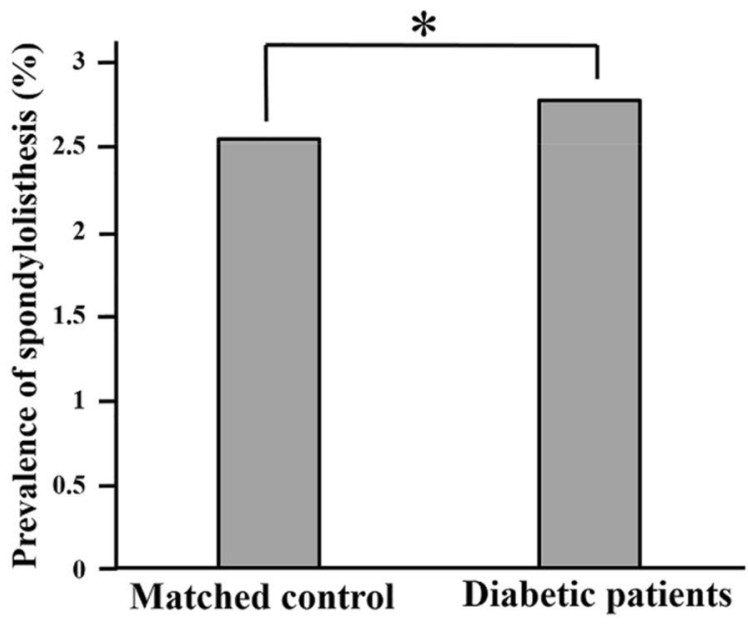

B

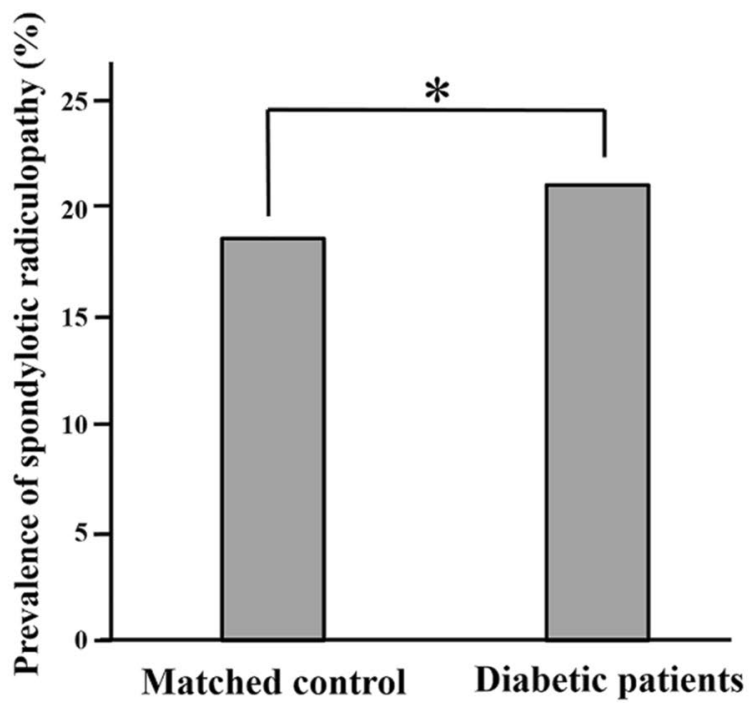

D

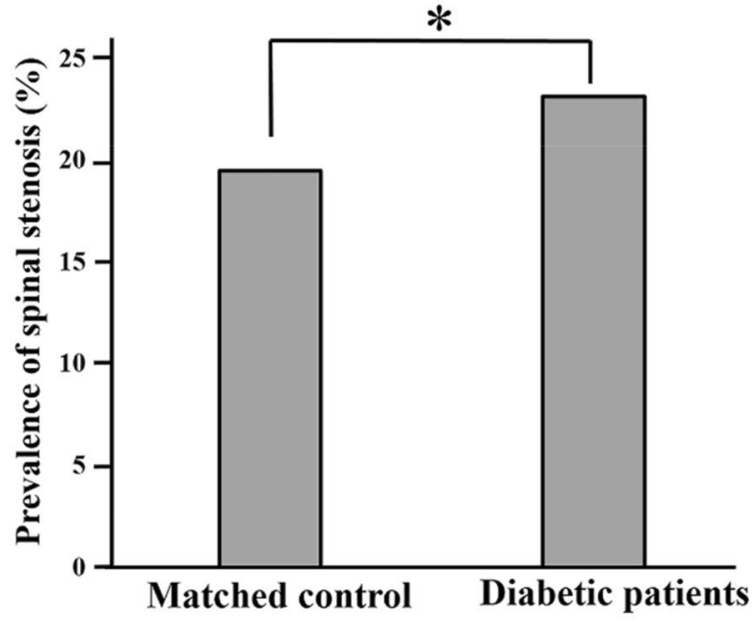

Figure 2. Prevalence of (A) lumbar disc disorder, (B) spondylotic radiculopathy, (C) spondylolisthesis, (D) spinal stenosis in matched control and diabetic patients. ${ }^{*} p<0.0001$. 
A

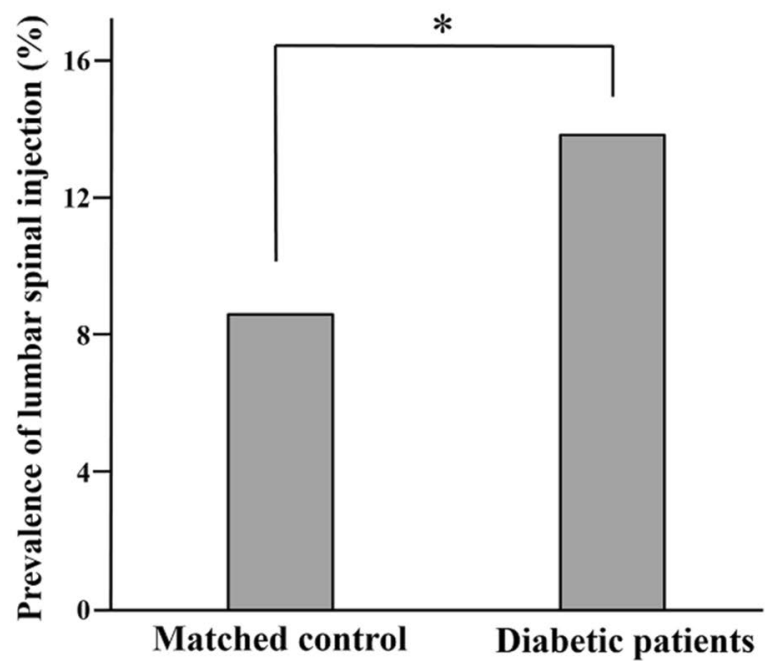

B

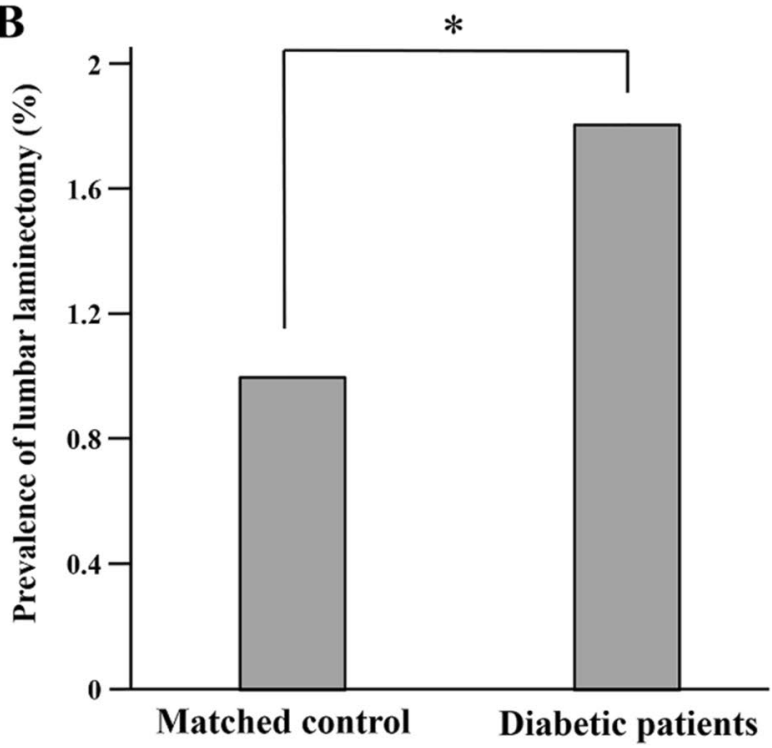

C

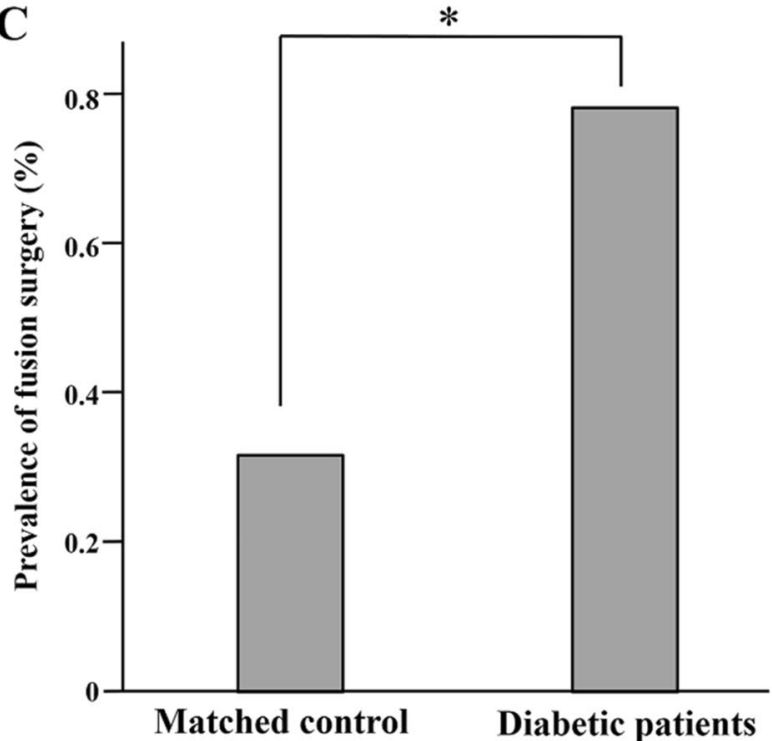

Figure 3. Prevalence of (A) lumbar spinal injection, (B) lumbar laminectomy, and (C) fusion operation in matched control and diabetic patients. ${ }^{\star} p<0.0001$.

Subgroup analysis according to diabetic complication. In subgroup analysis of diabetic complication, patients with complicated diabetes had a significantly higher risk of lumbar disc disorder (aOR 1.40, 95\% CI 1.38-1.42) and spondylotic radiculopathy (aOR 1.37, 95\% CI 1.35-1.39) compared with uncomplicated diabetes (Fig. 4A). Complicated diabetes had an increased risk of lumbar spondylolisthesis (aOR 1.31, 95\% CI 1.27-1.36) and spinal stenosis (aOR 1.59, 95\% CI 1.56-1.61), respectively. Complicated diabetes was strongly associated with undergoing lumbar spinal injection (aOR 1.42, 95\% CI 1.39-1.44), lumbar laminectomy (aOR 1.54, 95\% CI 1.48-1.61), and fusion surgery (aOR 1.70, 95\% CI 1.59-1.81), respectively (Fig. 4B).

The associations of individual diabetic complication with lumbar spine disorders and spinal procedures were evaluated as referenced to uncomplicated diabetes (Supplementary Figs. S1-S4). Diabetic neuropathy was at highest risk for lumbar spinal disorders and spinal procedures. Retinopathy and arthropathy were highly associated with lumbar spine disorders and spinal injection. However, nephropathy was negatively associated with lumbar spine disorders and procedures.

\section{Discussion}

The present study revealed that type 2 diabetes was strongly associated with lumbar spine disorders including lumbar disc disorder, lumbar spondylotic radiculopathy, spondylolisthesis, and spinal stenosis. Furthermore, patients with diabetes exhibited higher risks of undergoing lumbar injections and surgical procedures, suggesting 


\begin{tabular}{|l|l|l|l|}
\hline Outcome & Groups & Crude OR (95\% CI) & Adjusted ${ }^{*}$ OR (95\% CI) \\
\hline \multirow{4}{*}{ Lumbar disc disorder } & Matched control & Reference & Reference \\
\cline { 2 - 4 } & Diabetic patients & $1.15(1.14-1.17)$ & $1.11(1.10-1.12)$ \\
\cline { 2 - 4 } & $p$ value & $<0.0001$ & $<0.0001$ \\
\hline \multirow{4}{*}{ Spondylotic radiculopathy } & Matched control & Reference & Reference \\
\cline { 2 - 4 } & Diabetic patients & $1.17(1.16-1.18)$ & $1.12(1.11-1.13)$ \\
\cline { 2 - 4 } & $p$ value & $<0.0001$ & $<0.0001$ \\
\hline \multirow{3}{*}{ Spinal stenosis } & Matched control & Reference & Reference \\
\cline { 2 - 4 } & Diabetic patients & $1.10(1.06-1.12)$ & $1.05(1.02-1.08)$ \\
\cline { 2 - 4 } & $p$ value & $<0.0001$ & $<0.0001$ \\
\hline & Matched control & Reference & Reference \\
\cline { 2 - 4 } & Diabetic patients & $1.24(1.23-1.26)$ & $1.16(1.15-1.18)$ \\
\cline { 2 - 4 } & $p$ value & $<0.0001$ & $<0.0001$ \\
\hline
\end{tabular}

Table 2. Association of type 2 diabetes with lumbar disc disorder, spondylotic radiculopathy spondylolisthesis, and spinal stenosis. OR Odds ratio, CI Confidence interval. ${ }^{\star}$ Adjusted by age, sex, type of beneficiary, hypertension, chronic kidney disease, and dyslipidemia.

\begin{tabular}{|l|l|l|l|}
\hline Outcome & Groups & Crude OR $\mathbf{( 9 5 \% ~ C I ) ~}$ & Adjusted $^{*}$ OR $\mathbf{( 9 5 \% ~ C I ) ~}$ \\
\hline \multirow{4}{*}{ Spinal injection } & Matched control & Reference & Reference \\
\cline { 2 - 4 } & Diabetic patients & $1.19(1.17-1.20)$ & $1.13(1.12-1.14)$ \\
\cline { 2 - 4 } & $p$ value & $<0.0001$ & $<0.0001$ \\
\hline \multirow{4}{*}{ Lumbar laminectomy } & Matched control & Reference & Reference \\
\cline { 2 - 4 } & Diabetic patients & $1.26(1.22-1.30)$ & $1.19(1.15-1.23)$ \\
\cline { 2 - 4 } & $p$ value & $<0.0001$ & $<0.0001$ \\
\hline \multirow{3}{*}{ Lumbar fusion } & Matched control & Reference & Reference \\
\cline { 2 - 4 } & Diabetic patients & $1.48(1.40-1.56)$ & $1.35(1.29-1.42)$ \\
\cline { 2 - 4 } & $p$ value & $<0.0001$ & $<0.0001$ \\
\hline
\end{tabular}

Table 3. Association of type 2 diabetes with the prevalence of lumbar spinal injection, laminectomy, and lumbar fusion. OR Odds ratio, CI Confidence interval. *Adjusted by age, sex, type of beneficiary, hypertension, chronic kidney disease, and dyslipidemia.

diabetes is closely related to aggravating lumbar spine conditions, which ultimately necessitates more spinal procedures. Among diabetic patients, diabetic complication was presented as a significant risk factor for lumbar spine disorders and frequent spinal intervention. To the best of our knowledge, this is the first nationwide epidemiological evidence of associations of type 2 diabetes with major lumbar spine disorders and spinal procedures. This study had the following strengths: large sample size with matched control subjects, reliable disease diagnosis purely by physicians, and minimized effect of socioeconomic status under the National Health Insurance system.

Association of type 2 diabetes mellitus and degenerative lumbar spine disorders. Close relationship between diabetes and lumbar spine disorders has long been recognized in animal studies. Diabetic models showed several deleterious changes such as increasing toxic end products of glycation (advance glycation end products, AGEs), expression of matrix metalloproteinases (MMP)-2 related to degradation of extracellular matrix, and hyperglycemia-induced inflammation in intervertebral disc, which promotes intervertebral disc degeneration process ${ }^{15-17,24-26}$. However, despite the mechanisms for diabetes-related pathway demonstrated in animal studies, clinical evidence for the relationship between diabetes and intervertebral disc degeneration remains still lacking ${ }^{27}$. There are some clinical studies that chronic back pain was associated with type 2 diabetes, findings in line with our study ${ }^{19,20,28}$. Eivazi et al. demonstrated that the prevalence of chronic low back pain were $63.4 \%$ in diabetic adults and $47.0 \%$ in non-diabetic adults ${ }^{19}$. With respect to degenerative spine disorders, Agius et al. presented that the lower height of lumbar discs was significantly associated with type 2 diabetes, suggesting that degenerative disc disorders is closely related to diabetes ${ }^{22}$. In contrast, Fabiane et al. reported that there was no significant association between diabetes and lumbar disc disorder in twin volunteers ${ }^{23}$. One recent study has raised a possible connection between diabetes and spinal stenosis, revealing a high frequency of diabetes in patients with spinal stenosis ${ }^{29}$. Previous clinical studies for relation between diabetes and lumbar spine disorders have included only a small number of patients and was inconclusive. Therefore, this study being a large-population study is important in which it confirms the relationship between type 2 diabetes and the major lumbar spine disorders such as lumbar disc disorder, spondylotic radiculopathy, spondylolisthesis, and spinal stenosis. 


\section{Uncomplicated DM vs Complicated DM}

A

Lumbar disc disorder

Spondylotic radiculopathy

Spondylolisthesis

Adjusted OR (95\% CI)

$1.40(1.38-1.42)$

$1.37(1.35-1.39)$

$1.31(1.27-1.36)$

Spinal stenosis

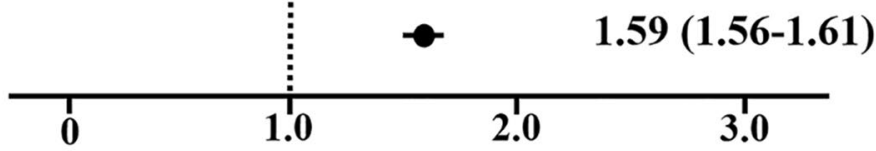

B

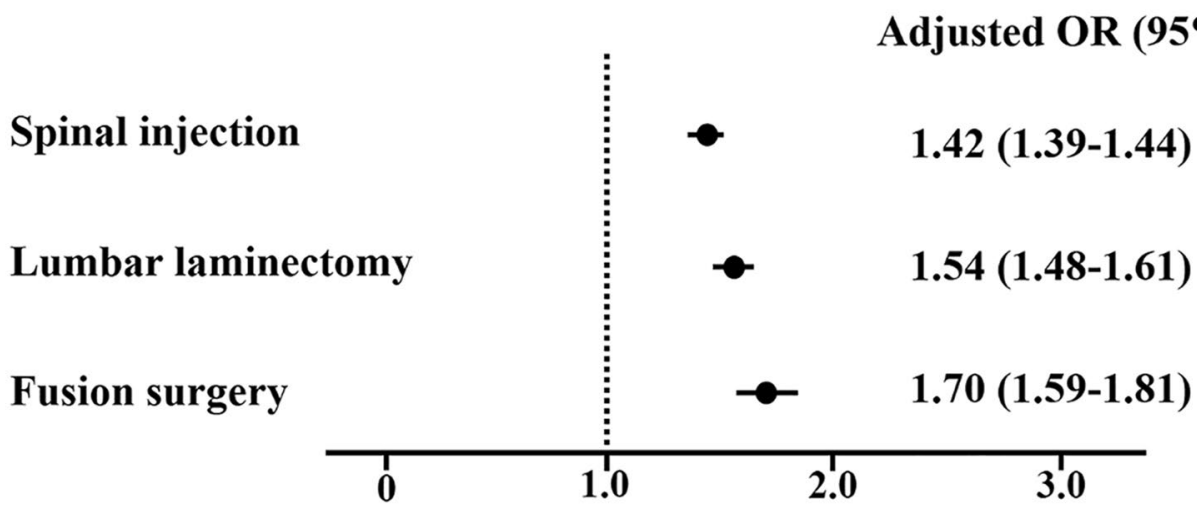

Figure 4. Association of complicated diabetes with (A) lumbar spine disorders and (B) spinal procedures compared with uncomplicated diabetes. Adjusted OR (95\% CI) was estimated by multiple logistic regression analysis with adjustments for hypertension, chronic kidney disease, dyslipidemia, and the type of beneficiary. $\mathrm{DM}$, diabetes mellitus; OR, odds ratio; $\mathrm{CI}$, confidence interval.

Diabetes associated with increased prevalence of lumbar spinal procedures. We firstly report the impact of diabetes on the prevalence of lumbar spine procedures, suggesting diabetes be recognized as a possible contributing factor of aggravating lumbar spine disorders. This study presented that diabetic patients underwent more lumbar surgeries than those without diabetes. Furthermore, diabetes was suggested as a potential risk factor for increasing lumbar surgery rates including lumbar laminectomy with/without discectomy, and fusion surgery. The indications for these lumbar surgeries were herniated lumbar disc disorder with neurologic signs of lumbar laminectomy with/without discectomy, and spinal instability, advanced spondylolisthesis, or spinal stenosis for lumbar fusion operation. Because these indications are the most critical value in clinical field for patients with degenerative lumbar spine disorder, we focused on these lumbar surgeries in consideration of those patients having a severe surgery-indicated status of their lumbar spine. Therefore, the present finding that diabetes was significantly associated with increased lumbar spine surgeries is of critical importance, since this suggests that diabetes can be a predisposing factor for increasing the severity of lumbar spine disorders, leading ultimately to the necessity of a surgical procedure.

Association of diabetic complication with lumbar spine condition. We showed that diabetic complication significantly associated with increased prevalence of lumbar spine disorders and spinal procedures compared with uncomplicated diabetes. Although these complications might exist at the time of the clinical diagnosis, the prevalence of diabetic complications increases over time. Furthermore, uncontrolled hyperglycemia may accelerate the progression of these complications. Thus, the rationale for regular health-checkups is that the frequency of the diabetic complications, especially vascular complications, increases with disease duration ${ }^{30}$. Thus, it is possible to assume that the status of diabetic patients with complications may be categorized as longstanding or uncontrolled diabetes ${ }^{14,31}$. In a recent study $^{21}$, a longer duration of type 2 diabetes was suggested 
to be a risk factor for lumbar disc degeneration, as shown by a cross-sectional study using magnetic resonance imaging (MRI), a result which is in line with our findings. Because diabetic complication is highly related to hyperglycemia and long-term suffering with diabetes ${ }^{32,33}$, this could produce toxic byproducts and inflammatory cytokines which could give harmful consequences for lumbar spine. Thus, long-term suffering from diabetes can aggravate lumbar spine condition, which possibly ends up necessitating lumbar spine procedures. Therefore, the present findings of the close relationship between complicated diabetes and lumbar spine disorders suggest physicians should take monitoring glycemic control more into consideration when treating back pain patients with a history of diabetes.

Which diabetic complications are highly associated with lumbar spine disorders and procedures? Multiple diabetic complications were included in this study to find out which complication is most associated with lumbar spine disorders and spine procedures. Of the many diabetes related complications, microvascular complications such as retinopathy, neuropathy, and nephropathy were included because these complications are of importance for the prognosis of diabetes ${ }^{23,33}$. Furthermore, diabetic arthropathy, being closely related to diabetic neuropathy was added to the analysis. Of these complications, diabetic neuropathy and arthropathy were the most highly associated lumbar spine disorders. These forms of diabetes, either neuropathy or arthropathy, had a high risk of more spine intervention and surgeries. Although arthropathy has multifactorial mechanical and vascular causes, arthropathy is known to be caused by deficit in proprioception secondary to diabetic neuropathy ${ }^{34}$. It is also called as neuropathic arthropathy or Charcot arthropathy. Therefore, diabetic neuropathy would be a key contributing factor associated with aggravating lumbar spine disorders. There are a several possible explanations. First, diabetic neuropathy increases the susceptibility of nerves to ischemic changes $^{35,36}$. These susceptible nerves can be trunks, roots, or peripheral nerves. One of important pathogenesis of radicular pain secondary to herniated lumbar disc disorders is mechanical compression of nerve roots and chemical irritations, thus having intra-neural edema formation and ischemic insults nerve roots ${ }^{35,37}$. Therefore, under conditions of diabetic neuropathy, the damage in the nerve root could be accelerated, possibly becoming more severe radicular pain in a herniated lumbar disc disorder. Second, mechanical sensitivity can be increased in afferent fibers of diabetic nerves ${ }^{38-40}$. Because diabetes nerves are known to have damaged myelinated and unmyelinated afferents, spontaneous aberrant activities from nociceptive C-fibers in diabetic nerves has been reported $^{40}$. These abnormal hyperactivities of nerves are susceptible to mechanical stimulation ${ }^{38,39}$. Approximately $97 \%$ of low back pain is thought to be mechanical origin pain ${ }^{2}$. Therefore, the hypersensitive state of diabetic nerve roots evoked by mechanical stimulation could be highly associated with painful lumbar spine disorders, thus having more spine interventions and surgeries in diabetic neuropathy.

The present study showed that diabetic retinopathy was a significant complication associated with increasing prevalence of lumbar spine disorders, though not as strongly as diabetic neuropathy or arthropathy. On the other hand, diabetic nephropathy was found not to be associated with increasing prevalence of lumbar spine disorders. Diabetic nephropathy is a well-known late-onset complication of diabetes, which would tend to imply a positive correlation with lumbar spine disorders. There are a several explanations for why this is not the case. First, although retinopathy, neuropathy, and nephropathy are microvascular complications, the relationship among the complications can be different. Recent systemic review and meta-analysis ${ }^{41}$ which presented the associations between the diabetic complications, showed that retinopathy was significantly associated with nephropathy and neuropathy, respectively. However, nephropathy had no association with neuropathy. Thus, even while neuropathy was shown to be closely related to lumbar spine disorders in this study, it may well be that nephropathy possesses different pathways, when compared to neuropathy. Second, the subjects of this study were sampled largely from inpatient populations. Of diabetic complications, nephropathy is a very long-standing and advanced complication. Thus, subjects with a history of admission, who were included in this study, may be admitted due to their serious medical problems. These patients' lumbar spine condition may not be considered due to an inability to undergo spine interventions and surgical procedures on them. Given this, lumbar spine disorders would be under-diagnosed in patients with diabetic nephropathy.

Limitations. There are a few limitations. First, we used national insurance claim data which can have fail to include prescriptions outside of insurance coverage (though this is less than $1 \%$ in South Korea), included possible incorrect diagnosis codes, and misclassification errors. But, due to the large-sample population, we believe such errors had little effect on our results. The major strength of this study is that it is a representative result drawn from a nationwide database of the South Korean population. Thus, this minimizes recruitment bias, or selection bias, something which is unavoidable in a cohort study. Second, although we have demonstrated that diabetic neuropathy was associated with lumbar spine disorders, clinical differentiation of lumbar radiculopathy from the diabetic radiculopathy is difficult due to similar clinical presentations between the diseases. Thus, the over-estimation of the association between neuropathy and lumbar spine disorders might be concerned. Nevertheless, the two diseases can be differentiated by electromyography or some different clinical features ${ }^{42}$. Because the diagnosis of the diseases was solely dependent on physician decision in this study, the misclassification errors are expected to be minimized. Third, there can be a selection bias. Patients with diabetes would be more likely to visit the hospital than those without diabetes. It is possible that screening for lumbar spine disorder could be conducted earlier in diabetic patients. Lastly, this study is a retrospective, cross-sectional study that cannot confirm the causal relationship. Therefore, a future study is still needed to find out the predictive effect of diabetes on degenerative lumbar spine disorders. Furthermore, we should investigate whether poor glycemic control in patients with diabetes would affect the lumbar spine condition in a prospective cohort study. 


\section{Conclusions}

In conclusion, this current, nationwide population-based study demonstrated that type 2 diabetes is associated with increased risks of comorbid lumbar spine disorders and undergoing spinal procedures. Furthermore, patients with complicated diabetes had greater risks for lumbar spine disorders and frequent spinal procedures, which confirms the association between type 2 diabetes and lumbar spinal diseases. This work suggests that diabetes may be a predisposing factor for degenerative lumbar spine disorder, which highlights the importance of early detection for lumbar spine pathology in diabetic patients. Future long-term prospective studies are warranted to investigate the underlying pathophysiological mechanism.

\section{Materials and methods}

Data source and study subjects. We conducted a 3-year population-based, matched case-control study using the 2016 to 2018 claims database of the Health Insurance Review and Assessment Service-National Inpatient Sample (HIRA-NIS). The Korean National Health Insurance Service is a single payer social insurance system with compulsory enrollment, in which the raw claims dataset of the HIRA includes almost all Korean citizens, approximately 46 million population ${ }^{43}$. HIRA-NIS is a population-based representative annual sample of one million patients' health data covering the entirety of all regions of South Korea ${ }^{43}$. This database utilizes a probabilistic weighted sample extraction method (sampling rate: $13 \%$ of total inpatients population, $1 \%$ of total out-patients population), which can be converted to detail the entire Korean population. All clinics and hospitals in Korea must provide the HIRA with information on diagnosis, treatment, and surgery.

The HIRA claims for inpatient and outpatient diagnoses, treatments and surgical procedures are coded using the International Statistical Classification of Diseases and Related Health Problems, 10th revision (ICD-10), and the Korean Drug and Anatomical Therapeutic Chemical Codes ${ }^{44,45}$. The HIRA regularly audits the database, which have been used in a variety of peer-review publications. The data derived from the database is thus considered reliable ${ }^{43,46}$.

Based on a definition of diabetes from a previous study ${ }^{45}$, patients with type 2 diabetes were defined as showing the presence of identical E11-E14 (ICD-10) codes at least two times, or an E11-E14 code and the prescription of anti-diabetic medications, which entails the use of sulfonylurea, meglitinides, biguanides, thiazolidinediones, dipeptidyl peptidase- 4 inhibitors, a-glucosidase inhibitors, sodium-glucose co-transporter 2 inhibitors, glucagon-like peptide 1 agonists, or insulin. Furthermore, the data was searched again to identify patients who had complications related to diabetes for a subgroup analysis. These subjects were included if they had any of the following diagnoses of diabetic complications: diabetic retinopathy (diagnosis code: H360), diabetic nephropathy (N083, E112, E122, E132, or E142), diabetic arthropathy (M142 or M146), and diabetic neuropathy (G590, G632, or G990) $)^{47,48}$.

Among the participants aged 20-89 years from HIRA database between January 2016 and December 2018 $(n=2,761,984)$, patients with type 2 diabetes were selected as the definition (Fig. 1$)$. The prevalence of type 2 diabetes was $17.4 \%$. For the control group, subjects were enrolled by using age- and sex- matched sampling methods that incorporate the propensity score at a 1:1 ratio of controls to diabetic patients.

Outcome measures. The disorders of interest were degenerative lumbar spine diseases, which are major causes of mechanical low back pain. Based on the previous studies ${ }^{49,50}$, the HIRA national database was searched to identify patients who had a primary diagnosis of (i) degenerative lumbar disc disorder (diagnosis code: M51, M512, M513, M518, M519), (ii) degenerative or spondylotic lumbar radiculopathy (M472, M511, or M541), (iii) degenerative lumbar spondylolisthesis (M431), or (iv) degenerative lumbar spinal stenosis (M48 or M995) were identified. These subjects were selected if they had undergone any of the spinal injections including lumbar epidural steroid injection (procedure code: LA322, HA102), selective nerve root injection (LA352-LA357), and facet or perifacet joint injection (LA358-LA359). Furthermore, the subjects were selected if they had any of the following primary spinal surgeries: (i) lumbar laminectomy with or without discectomy (procedure code: N1493, N14930, N1499, or N2499), and (ii) lumbar fusion operation (N2470, N1460, N1466, N1469, N0466, or N0469).

Confounding factors used in this study included type of beneficiary, hypertension (I10-I13), chronic kidney disease (N18), and dyslipidemia (E78).

Indications of lumbar spine surgeries. Surgical indications of degenerative lumbar disc disorder, spondylotic radiculopathy, spondylolisthesis, and spinal stenosis are noted by the National Health Insurance Corporation (NHIC) of the South Korea ${ }^{50,51}$. Almost all clinics and hospitals in South Korea have adopted the recording requirements of lumbar spine surgery, notifying the NHIC for financial reimbursement. The standard of surgical treatment for subjects with lumbar disc disorder and spondylotic radiculopathy is lumbar laminectomy, with or without discectomy, in patients with neurologic sign (e.g. numbness, paresthesia, or weakness), or chronic intractable pain despite having received conservative treatment for a period of time exceeding 3 months. For lumbar spondylolisthesis and spinal stenosis, a lumbar fusion operation is indicated, if lumbar spine instability or foraminal stenosis is present. Therefore, these requirements of the Korean NHIC are regarded as surgical indications for patients.

Statistical analysis. Prevalence was estimated as the number of cases divided by the study population. To compare the prevalence of outcomes between the diabetes and matched control groups, chi-square test was used. To determine the association of type 2 diabetes with the lumbar spine disorders and spinal procedures, a multiple logistic regression analysis was performed with adjustments for hypertension, chronic kidney disease, dyslipidemia, and the type of beneficiary. Adjusted odds ratios (aOR) were estimated to determine the 
association of diabetes with lumbar spine disorders and spinal procedures compared with the matched control group. Furthermore, a subgroup analysis was performed by the presence of diabetic complication. All statistical analyses, including analyses of the matching data, were performed using SAS software (SAS Institute, Inc., Cary, North Carolina). A $p$ value below 0.05 was considered statistically significant.

Ethics approval. Ethics approval for the study protocol and data analysis was obtained from the Institutional Review Board (IRB) of Seoul National University Hospital, Seoul, Republic of Korea (IRB number: E-2001-0011090). This study was conducted in accordance with the 1975 Declaration of Helsinki. Informed consent was waived by the Institutional Review Board of Seoul National University Hospital because the researchers retrospectively assessed de-identified data for analytical purposes.

\section{Data availability}

The datasets analyzed for the study are available from the corresponding author on reasonable request.

Received: 18 February 2021; Accepted: 28 May 2021

Published online: 13 August 2021

\section{References}

1. Lozano, R. et al. Measuring progress from 1990 to 2017 and projecting attainment to 2030 of the health-related Sustainable Development Goals for 195 countries and territories: A systematic analysis for the Global Burden of Disease Study 2017. Lancet 392, 2091-2138. https://doi.org/10.1016/S0140-6736(18)32281-5 (2018).

2. Deyo, R. A. \& Weinstein, J. N. Low back pain. N. Engl. J. Med. 344, 363-370. https://doi.org/10.1056/NEJM200102013440508 (2001).

3. Singh, K. \& Phillips, F. M. The Biomechanics and Biology of the Spinal Degenerative Cascade. Semin Spine Surg. 17, $128-136$. https://doi.org/10.1053/j.semss.2005.06.001 (2005).

4. Tobert, D. G. \& Harris, M. B. Degenerative lumbar spinal stenosis and spondylolisthesis. In Principles of Orthopedic Practice for Primary Care Providers 47-59 (Springer). https://doi.org/10.1007/978-3-319-68661-5_4 (2018).

5. Cohen, S. P., Argoff, C. E. \& Carragee, E. J. Management of low back pain. BMJ 337, a2718. https://doi.org/10.1136/bmj.a2718 (2008).

6. Huang, T. J., Sayers, N. M., Fernyhough, P. \& Verkhratsky, A. Diabetes-induced alterations in calcium homeostasis in sensory neurones of streptozotocin-diabetic rats are restricted to lumbar ganglia and are prevented by neurotrophin-3. Diabetologia 45, 560-570. https://doi.org/10.1007/s00125-002-0785-x (2002).

7. Kääriä, S. et al. Risk factors of sciatic pain: A prospective study among middle-aged employees. Eur. J. Pain 15, 584-590. https:// doi.org/10.1016/j.ejpain.2010.11.008 (2011).

8. Rubin, D. I. Epidemiology and risk factors for spine pain. Neurol. Clin. 25, 353-371. https://doi.org/10.1016/j.ncl.2007.01.004 (2007).

9. Taylor, J. B., Goode, A. P., George, S. Z. \& Cook, C. E. Incidence and risk factors for first-time incident low back pain: A systematic review and meta-analysis. Spine J. 14, 2299-2319. https://doi.org/10.1016/j.spinee.2014.01.026 (2014).

10. Hadjipavlou, A., Tzermiadianos, M., Bogduk, N. \& Zindrick, M. The pathophysiology of disc degeneration: A critical review. J. Bone Joint Surg. Br. 90, 1261-1270. https://doi.org/10.1302/0301-620X.90B10.20910 (2008).

11. Dario, A. B. et al. The relationship between obesity, low back pain, and lumbar disc degeneration when genetics and the environment are considered: A systematic review of twin studies. Spine J. 15, 1106-1117. https://doi.org/10.1016/j.spinee.2015.02.001 (2015).

12. Li, Z. et al. Clinical characteristics and risk factors of recurrent lumbar disk herniation: A retrospective analysis of three hundred twenty-one cases. Spine (Phila Pa 1976) 43, 1463-1469. https://doi.org/10.1097/BRS.0000000000002655 (2018).

13. Shiri, R. et al. Risk factors for low back pain: a Population-Based longitudinal study. Arthritis Care. Res. (Hoboken) 71, $290-299$. https://doi.org/10.1002/acr.23710 (2019).

14. Root, H. F., Pote, W. H. \& Frehner, H. Triopathy of diabetes: sequence of neuropathy, retinopathy, and nephropathy in one hundred fifty-five patients. AMA Arch. Intern. Med. 94, 931-941. https://doi.org/10.1001/archinte.1954.00250060065006 (1954).

15. Fields, A. J. et al. Alterations in intervertebral disc composition, matrix homeostasis and biomechanical behavior in the UCDT2DM rat model of type 2 diabetes. J. Orthop. Res. 33, 738-746. https://doi.org/10.1002/jor.22807 (2015).

16. Illien-Junger, S. et al. Combined anti-inflammatory and anti-AGE drug treatments have a protective effect on intervertebral discs in mice with diabetes. PLOS ONE 8, e64302. https://doi.org/10.1371/journal.pone.0064302 (2013).

17. Illien-Jünger, S. et al. Chronic ingestion of advanced glycation end products induces degenerative spinal changes and hypertrophy in aging pre-diabetic mice. PLoS ONE 10, e0116625. https://doi.org/10.1371/journal.pone.0116625 (2015).

18. Ritzwoller, D. P., Crounse, L., Shetterly, S. \& Rublee, D. The association of comorbidities, utilization and costs for patients identified with low back pain. BMC Musculoskelet. Disord. 7, 72. https://doi.org/10.1186/1471-2474-7-72 (2006).

19. Eivazi, M. \& Abadi, L. Low back pain in diabetes mellitus and importance of preventive approach. Health Promot. Perspect. 2, 80-88. https://doi.org/10.5681/hpp.2012.010 (2012).

20. Hassoon, A. et al. Chronic low-back pain in adult with diabetes: NHANES 2009-2010. J. Diabetes Complicat. 31, 38-42. https:// doi.org/10.1016/j.jdiacomp.2016.10.025 (2017).

21. Liu, X., Pan, F., Ba, Z., Wang, S. \& Wu, D. The potential effect of type 2 diabetes mellitus on lumbar disc degeneration: A retrospective single-center study. J. Orthop. Surg. Res. 13, 52. https://doi.org/10.1186/s13018-018-0755-8 (2018).

22. Agius, R., Galea, R. \& Fava, S. Bone mineral density and intervertebral disc height in type 2 diabetes. J. Diabetes Complicat. 30, 644-650. https://doi.org/10.1016/j.jdiacomp.2016.01.021 (2016).

23. Fabiane, S. M., Ward, K. J., Iatridis, J. C. \& Williams, F. M. Does type 2 diabetes mellitus promote intervertebral disc degeneration?. Eur. Spine J. 25, 2716-2720. https://doi.org/10.1007/s00586-016-4612-3 (2016).

24. Cheng, X. et al. Polyol pathway mediates enhanced degradation of extracellular matrix via p38 MAPK activation in intervertebral disc of diabetic rats. Connect. Tissue Res. 54, 118-122. https://doi.org/10.3109/03008207.2012.754886 (2013).

25. Chen, S., Liao, M., Li, J., Peng, H. \& Xiong, M. The correlation between microvessel pathological changes of the endplate and degeneration of the intervertebral disc in diabetic rats. Exp. Ther. Med. 5, 711-717. https://doi.org/10.3892/etm.2012.868 (2013).

26. Won, H.-Y., Park, J.-B., Park, E.-Y. \& Riew, K. D. Effect of hyperglycemia on apoptosis of notochordal cells and intervertebral disc degeneration in diabetic rats. J. Neurosurg. Spine 11, 741-748. https://doi.org/10.3171/2009.6.SPINE09198 (2009).

27. Alpantaki, K., Kampouroglou, A., Koutserimpas, C., Effraimidis, G. \& Hadjipavlou, A. Diabetes mellitus as a risk factor for intervertebral disc degeneration: A critical review. Eur. Spine J. 28, 2129-2144. https://doi.org/10.1007/s00586-019-06029-7 (2019).

28. Hinyard, L. J. \& Geremakis, C. M. Assessing health related quality of life in persons with diabetes: A comparison of generic measures. Open Public Health J. https://doi.org/10.2174/1874944501609010065 (2016). 
29. Asadian, L., Haddadi, K., Aarabi, M. \& Zare, A. Diabetes mellitus, a new risk factor for lumbar spinal stenosis: A case-control study. Clin. Med. Insights Endocrinol. Diabetes 9, 1-5. https://doi.org/10.4137/cmed.s39035 (2016).

30. Won, J. et al. Prevalence and clinical characteristics of diabetic peripheral neuropathy in hospital patients with type 2 diabetes in Korea. Diabet. Med. 29, e290-e296. https://doi.org/10.1111/j.1464-5491.2012.03697.x (2012).

31. Amos, A. F., McCarty, D. J. \& Zimmet, P. The rising global burden of diabetes and its complications: Estimates and projections to the year 2010. Diabet. Med. 14, S7-S85. https://doi.org/10.1002/(SICI)1096-9136(199712)14:5+\%3cS7::AID-DIA522\%3e3.0. CO;2-R (1997).

32. King, G. L. The role of inflammatory cytokines in diabetes and its complications. J. Periodontol. 79, 1527-1534. https://doi.org/ 10.1902/jop.2008.080246 (2008).

33. Williams, M. D. \& Nadler, J. L. Inflammatory mechanisms of diabetic complications. Curr. Diabetes Rep. 7, 242-248. https://doi. org/10.1007/s11892-007-0038-y (2007).

34. Brower, A. \& Allman, R. Pathogenesis of the neurotrophic joint: Neurotraumatic vs. neurovascular. Radiology 139, 349-354. https:// doi.org/10.1148/radiology.139.2.7220879 (1981).

35. Takahashi, N., Yabuki, S., Aoki, Y. \& Kikuchi, S. Pathomechanisms of nerve root injury caused by disc herniation: An experimental study of mechanical compression and chemical irritation. Spine (Phila Pa 1976) 28, 435-441. https://doi.org/10.1097/01.BRS.00000 48645.33118.02 (2003).

36. Yabuki, S., Igarashi, T. \& Kikuchi, S. Application of nucleus pulposus to the nerve root simultaneously reduces blood flow in dorsal root ganglion and corresponding hindpaw in the rat. Spine (Phila Pa 1976) 25, 1471-1476. https://doi.org/10.1097/00007632-20000 6150-00004 (2000)

37. Rydevik, B., Brown, M. D. \& Lundborg, G. Pathoanatomy and pathophysiology of nerve root compression. Spine (Phila Pa 1976) 9, 7-15. https://doi.org/10.1097/00007632-198401000-00004 (1984).

38. Khan, G., Chen, S.-R. \& Pan, H.-L. Role of primary afferent nerves in allodynia caused by diabetic neuropathy in rats. Neuroscience 114, 291-299. https://doi.org/10.1016/s0306-4522(02)00372-x (2002).

39. Chen, X. \& Levine, J. Hyper-responsivity in a subset of C-fiber nociceptors in a model of painful diabetic neuropathy in the rat. Neuroscience 102, 185-192. https://doi.org/10.1016/s0306-4522(00)00454-1 (2001).

40. Ahlgren, S. C., White, D. M. \& Levine, J. Increased responsiveness of sensory neurons in the saphenous nerve of the streptozotocindiabetic rat. J. Neurophysiol. 68, 2077-2085. https://doi.org/10.1152/jn.1992.68.6.2077 (1992).

41. Li, J. et al. Correlations among diabetic microvascular complications: A systematic review and meta-analysis. Sci. Rep. 9, 1-9. https://doi.org/10.1038/s41598-019-40049-z (2019)

42. Hirsh, L. F. Diabetic polyradiculopathy simulating lumbar disc disease: Report of four cases. J. Neurosurg. 60, 183-186. https:// doi.org/10.3171/jns.1984.60.1.0183 (1984).

43. Kim, L., Kim, J.-A. \& Kim, S. A guide for the utilization of health insurance review and assessment service national patient samples. Epidemiol. Health 36, e2014008. https://doi.org/10.4178/epih/e2014008 (2014).

44. Chun, C. B., Kim, S. Y., Lee, J. Y. \& Lee, S. Y. Republic of Korea: Health System Review. Vol. 11183 (World Health Organization Regional Office for Europe, 2009).

45. Hwangbo, Y. et al. Incidence of diabetes after cancer development: A Korean national cohort study. JAMA Oncol. 4, 1099-1105. https://doi.org/10.1001/jamaoncol.2018.1684 (2018).

46. Kim, J., Yoon, S., Kim, L.-Y. \& Kim, D.-S. Towards actualizing the value potential of Korea Health Insurance Review and Assessment (HIRA) data as a resource for health research: Strengths, limitations, applications, and strategies for optimal use of HIRA data. J. Korean Med. Sci. 32, 718-728. https://doi.org/10.3346/jkms.2017.32.5.718 (2017).

47. Metcalf, P. A., Kyle, C., Kenealy, T. \& Jackson, R. T. HbAlc in relation to incident diabetes and diabetes-related complications in non-diabetic adults at baseline. J. Diabetes Complicat. 31, 814-823. https://doi.org/10.1016/j.jdiacomp.2017.02.007 (2017).

48. Min, K. L. et al. Utilization patterns of insulin for patients with type 2 diabetes from national health insurance claims data in South Korea. PLoS ONE 14, e0210159. https://doi.org/10.1371/journal.pone.0210159 (2019).

49. Lee, C.-H., Chung, C. K., Kim, C. H. \& Kwon, J.-W. Health care burden of spinal diseases in the Republic of Korea: Analysis of a nationwide database from 2012 through 2016. Neurospine 15, 66. https://doi.org/10.14245/ns.1836038.019 (2018).

50. Park, M. S. et al. Repeat decompression and fusions following posterolateral fusion versus posterior/transforaminal lumbar interbody fusion for lumbar spondylosis: A national database study. Sci. Rep. 9, 4926. https://doi.org/10.1038/s41598-019-41366-z (2019).

51. Park, M. S. et al. Reoperation rates after anterior cervical discectomy and fusion for cervical spondylotic radiculopathy and myelopathy: A national population-based study. Spine (Phila Pa 1976) 41, 1593-1599. https://doi.org/10.1097/BRS.0000000000 001590 (2016)

\section{Author contributions}

C.-H.P.: manuscript drafting and study design. K.-B.M.: critical revision and data interpretation. J.-Y.M.: statistical analysis and study design. D.H.K. and K.M.S.: manuscript revision for important intellectual content. D.-K.K.: critical revision and main study design. All authors: final version approval.

\section{Funding}

This research received no external funding.

\section{Competing interests}

The authors declare no competing interests.

\section{Additional information}

Supplementary Information The online version contains supplementary material available at https://doi.org/ 10.1038/s41598-021-95626-y.

Correspondence and requests for materials should be addressed to D.-K.K.

Reprints and permissions information is available at www.nature.com/reprints.

Publisher's note Springer Nature remains neutral with regard to jurisdictional claims in published maps and institutional affiliations. 
(c) (i) Open Access This article is licensed under a Creative Commons Attribution 4.0 International cc) License, which permits use, sharing, adaptation, distribution and reproduction in any medium or format, as long as you give appropriate credit to the original author(s) and the source, provide a link to the Creative Commons licence, and indicate if changes were made. The images or other third party material in this article are included in the article's Creative Commons licence, unless indicated otherwise in a credit line to the material. If material is not included in the article's Creative Commons licence and your intended use is not permitted by statutory regulation or exceeds the permitted use, you will need to obtain permission directly from the copyright holder. To view a copy of this licence, visit http://creativecommons.org/licenses/by/4.0/.

(C) The Author(s) 2021 